\title{
Distinct neuronal patterns of positive and negative moral processing in psychopathy
}

\author{
Samantha J. Fede ${ }^{1,2}$ - Jana Schaich Borg ${ }^{3}$ - Prashanth K. Nyalakanti ${ }^{2}$. \\ Carla L. Harenski ${ }^{2}$ - Lora M. Cope ${ }^{4}$ - Walter Sinnott-Armstrong ${ }^{3}$ - Mike Koenigs ${ }^{5}$. \\ Vince D. Calhoun ${ }^{1,2}$ • Kent A. Kiehl ${ }^{1,2}$
}

Published online: 22 August 2016

(C) Psychonomic Society, Inc. 2016

\begin{abstract}
Psychopathy is a disorder characterized by severe and frequent moral violations in multiple domains of life. Numerous studies have shown psychopathy-related limbic brain abnormalities during moral processing; however, these studies only examined negatively valenced moral stimuli. Here, we aimed to replicate prior psychopathy research on negative moral judgments and to extend this work by examining psychopathy-related abnormalities in the processing of controversial moral stimuli and positive moral processing. Incarcerated adult males $(N=245)$ completed a functional magnetic resonance imaging protocol on a mobile imaging system stationed at the prison. Psychopathy was assessed using the Hare Psychopathy Checklist-Revised (PCL-R). Participants were then shown words describing three types of moral stimuli: wrong (e.g., stealing), not wrong (e.g., charity), and controversial (e.g., euthanasia). Participants rated each stimulus as either wrong or not wrong. PCL-R total scores were correlated with not wrong behavioral responses
\end{abstract}

Electronic supplementary material The online version of this article (doi:10.3758/s13415-016-0454-z) contains supplementary material, which is available to authorized users.

Samantha J. Fede

sjfede@unm.edu

$\triangle$ Kent A. Kiehl

kkiehl@mrn.org

University of New Mexico, Albuquerque, New Mexico

2 Mind Research Network, An Affiliate of Lovelace Biomedical and Environmental Research Institute, 1101 Yale Blvd NE, Albuquerque, New Mexico 87131

3 Duke University, Durham, NC, USA

4 University of Michigan, Ann Arbor, MI, USA

5 University of Wisconsin, Madison, WI, USA to wrong moral stimuli, and were inversely related to hemodynamic activity in the anterior cingulate cortex in the contrast of wrong $>$ not wrong. In the controversial $>$ noncontroversial comparison, psychopathy was inversely associated with activity in the temporal parietal junction and dorsolateral prefrontal cortex. These results indicate that psychopathy-related abnormalities are observed during the processing of complex, negative, and positive moral stimuli.

Keywords Psychopathy $\cdot$ Moral $\cdot$ Prosocial $\cdot$ Incarceration · fMRI $\cdot$ Decision making

Psychopathy is a clinical condition characterized by deficient emotional reactivity and antisocial traits (Hare, 2003). Psychopathic individuals regularly commit moral violations and are responsible for a disproportionate amount of violent and repetitive crime. Psychopaths also constitute approximately $25 \%$ of incarcerated populations (Alterman, Cacciola, \& Rutherford, 1993; Hare, 2003). These callous and antisocial behaviors contribute to a high financial burden, estimated to be $30 \%-50 \%$ of the $\$ 3.2$ trillion annual societal cost of crime in the United States (Anderson, 2012; Kiehl, 2014; Kiehl \& Hoffman, 2011).

The enormous impact of psychopathy on society has led a growing number of researchers to examine the underlying neurobiology related to the condition. In particular, studies have begun to examine psychopathy-related moral processing abnormalities with the goal of developing new treatments to remediate these problems. However, the picture of moral processing in psychopathy is incomplete. Initial clinical observations confirmed that immoral behaviors are more common among psychopaths than non-psychopaths (Cleckley, 1976). However, the literature is mixed as to whether or not psychopaths can correctly make moral judgments; experimental 
studies have found that psychopaths usually do not differ from non-psychopaths on tasks in which they classify stimuli as morally right or wrong (Aharoni, Sinnott-Armstrong, \& Kiehl, 2012; Cima, Tonnaer, \& Hauser, 2010; Glenn, Raine, \& Schug, 2009; Harenski, Harenski, Shane, \& Kiehl, 2010; O'Kane, Fawcett, \& Blackburn, 1996; Simon, Holzberg, \& Unger, 1951), although psychopaths do make more utilitarian moral judgments (Blair, 1995; Koenigs, Kruepke, Zeier, \& Newman, 2012; Young, Koenigs, Kruepke, \& Newman, 2012). Despite the similar capacities for moral judgment, psychopaths do show different patterns of brain engagement than nonpsychopaths when processing moral stimuli (Glenn, Raine, \& Schug, 2009; Harenski, Edwards, Harenski, \& Kiehl, 2014; Harenski et al., 2010). Psychopathy-related effects have been reported to include reduced activation in the amygdala, posterior cingulate (PCC), and temporal parietal junction (TPJ) during processing of moral stimuli. These aforementioned brain regions play important roles in moral judgment. It is posited that the amygdala and the vmPFC work through stimulus-reinforcement learning to associate distress with moral transgressions to reduce antisocial behaviors (Blair, 2007). The PCC is engaged when individuals use theory of mind to generate intent stories, during self-reflection processes, and when integrating emotion into moral decision making (Fletcher et al., 1995; Greene, Sommerville, Nystrom, Darley, \& Cohen, 2001; Johnson et al., 2006; Ochsner \& Gross, 2005).

The psychopathy studies summarized above have selectively examined moral judgment of negatively valenced stimuli (e.g., indicating the severity of the moral violation of hitting someone with a bat; Harenski et al., 2010). To date, no studies have investigated the neural correlates of positive moral judgment in psychopathy (e.g., whether or not giving to charity is morally wrong or not wrong). Research on other prosocial processes, such as social cooperation, has indicated that psychopathy is associated with reduced engagement of the orbitofrontal cortex (OFC), dorsolateral prefrontal cortex (dlPFC), and anterior cingulate cortex (ACC; Rilling et al., 2007). Additionally, structural magnetic resonance imaging analyses have indicated that psychopathy is associated with reduced grey matter in several paralimbic regions implicated in positive moral judgment in healthy subjects, including in the insula, PCC, amygdala, and ACC (Boccardi et al., 2011; de Oliveira-Souza et al., 2008; Ermer, Guerin, Cosmides, Tooby, \& Miller, 2006; Ermer \& Kiehl, 2010; Ly et al., 2012; Ma, Wang, \& Han, 2011; Rilling et al., 2008; Rilling et al., 2002; Schaich Borg, Sinnott-Armstrong, Calhoun, \& Kiehl, 2011; Tiihonen et al., 2008; Yang, Raine, Narr, Colletti, \& Toga, 2009). The paralimbic hypothesis suggests that characteristics of psychopathy, including affective and interpersonal traits as well as antisocial ones, are related to abnormal functioning and structure in limbic and connected structures (Kiehl, 2006). In this model, abnormal functioning in both attentional/goal-oriented frontal regions and socioaffective regions lead to a lack of attention to emotional cues and learning as well as poor planning and error monitoring. Deficiencies are thought to exist both in individual paralimbic regions (as we discussed above) and in the default mode and frontoparietal networks (Juárez, Kiehl, \& Calhoun, 2013).

Many studies of moral processing have focused on clearcut moral transgressions (i.e., using stimuli involving causing pain to others). Some studies of nonclinical populations have started to examine other components of moral processing, such as philosophical stimuli, intentionality in moral judgment, and charity scenarios (Greene et al., 2001; Rilling et al., 2008; Young, Camprodon, Hauser, Pascual-Leone, \& Saxe, 2010). Recently, we examined judgments of actions that are prosocial (e.g., sharing, friendship) or morally controversial (e.g., abortion or euthanasia), along with traditional clearcut moral violations (Schaich Borg et al., 2011). Hemodynamic activity related to moral judgment was observed in the insula, vmPFC, PCC, temporal poles, basal ganglia, TPJ, and amygdala. Additionally, the hemodynamic response was greater in response to morally controversial stimuli than to clear-cut moral stimuli.

In the present study, we used functional magnetic resonance imaging (fMRI) to investigate the neural correlates of processing wrong, not wrong, and controversial moral stimuli in psychopathy. We aimed to replicate prior psychopathyrelated findings of moral judgments related to moral transgressions (i.e., reduced amygdala, TPJ, and PCC activation related to psychopathy) and to extend the previous work by incorporating judgments of positive and controversial stimuli. To the authors' knowledge, this is the first study to examine the neural correlates of positive (i.e., not wrong) moral processing and psychopathy, and the first study to compare the neural correlates of complex (i.e., controversial) versus simple (i.e., noncontroversial) moral cognition in psychopathy.

Understanding of these potentially distinct moral processes in psychopathy may lead to novel rehabilitative approaches leading to reductions in the impact of a disorder currently responsible for billions of dollars of societal costs (Anderson, 2012; Kiehl, 2014; Kiehl \& Hoffman, 2011). For example, in a program at Mendota Juvenile Treatment Center, a secure facility in Wisconsin that houses many juvenile offenders who are high in psychopathic traits, a unique, positivereinforcement-based program is having highly positive outcomes where other treatment approaches have failed (Caldwell, McCormick, Umstead, \& Van Rybroek, 2007). Similarly, understanding how psychopaths process prosocial stimuli and controversial targets may provide an avenue for a cognitive based therapeutic approach to changing the mentality of offenders that lead to moral violations.

On the basis of previous work, we hypothesized that psychopathic traits would be inversely related to activity in 
regions engaged in healthy moral processing that have previously been found to be dysfunctional in psychopathy. Specifically, we expected that hemodynamic response in the amygdala, ACC, PCC, ventroanterior insula (vAI), and TPJ would be inversely related to PCL-R scores during the processing of wrong as compared to not wrong moral stimuli. Although the ACC and vAI have not been found specifically in moral processing studies of psychopathy, these regions are structurally connected to the amygdala (Price, 2003), implicated in healthy moral processing, structurally and functionally abnormal in psychopathy, and related to affective and emotion-processing deficits in psychopathy (Chang, Yarkoni, Khaw, \& Sanfey, 2013; Kiehl et al., 2001). On the basis of prior neuroimaging studies of psychopathy and positive social processes, we also predicted that psychopathy would be related to reduced engagement in the OFC, dIPFC, and ACC (Rilling et al., 2007), during the processing of not wrong moral stimuli. Finally, given the stronger hemodynamic response to controversial moral stimuli in the previous study, we expected that we would see more pronounced inverse effects of psychopathy related to controversial moral judgment in the aforementioned regions (the amygdala, ACC, PCC, vAI, TPJ, dIPFC, and OFC).

\section{Method and materials}

\section{Participants}

The participants were incarcerated adult males from prisons in New Mexico and Wisconsin $(N=245)$ in which we have established research programs. Participants provided written, informed consent and were compensated $\$ 1 / \mathrm{h}$, comparable to the pay for general labor work in the facilities. Their IQs ranged from 66 to 134, and their ages were between 18 and 65 (see Table 1 for demographics). The exclusion criteria were an English reading level below 4th grade, history of neurological disorder or stroke, head injury with loss of consciousness greater than $1 \mathrm{~h}$, or history of psychotic disorder in the self or in a first-degree relative. All procedures and materials were approved by the University of New Mexico Institutional Review Board, and participants' consent was obtained according to the Declaration of Helsinki.

\section{Assessments}

Psychopathy was assessed using the Hare Psychopathy Checklist-Revised (PCL-R), the gold standard of psychopathy in forensic populations (Hare, 2003). Trained researchers reviewed institutional records and conducted semistructured interviews covering topics including school and employment history, family and peer relations, antisocial behaviors, and interpersonal style. The PCL-R comprises 20 items, each
Table 1 Descriptive statistics for forensic sample: Demographics, IQ, psychopathy, and substance dependence $(N=245)$

\begin{tabular}{|c|c|c|c|}
\hline Variable & Mean & $S D$ & Percentage \\
\hline Age & 36.14 & 10.85 & \\
\hline \multicolumn{4}{|l|}{ Handedness } \\
\hline Right & & & 82.4 \\
\hline Left & & & 9.8 \\
\hline Ambidextrous & & & 6.9 \\
\hline \multicolumn{4}{|l|}{ Ethnicity/Race } \\
\hline Hispanic/Latino & & & 40.3 \\
\hline Not Hispanic/Latino & & & 59.7 \\
\hline American Indian/Alaskan Native & & & 14.0 \\
\hline Asian & & & 0.5 \\
\hline Black/African American & & & 10.0 \\
\hline Native Hawaiian/Pacific Islander & & & 0.0 \\
\hline White & & & 50.7 \\
\hline Other/Decline & & & 24.9 \\
\hline IQ & 96.85 & 14.06 & \\
\hline \multicolumn{4}{|l|}{ Psychopathy } \\
\hline Total & 20.73 & 6.81 & \\
\hline Factor 1 & 6.18 & 3.39 & \\
\hline Factor 2 & 12.19 & 3.90 & \\
\hline \multicolumn{4}{|l|}{ Substance Dependence } \\
\hline None & & & 35.5 \\
\hline Alcohol & & & 45.7 \\
\hline Sedatives & & & 4.9 \\
\hline Cannabis & & & 26.2 \\
\hline Methamphetamine & & & 22.5 \\
\hline Opioids & & & 18.0 \\
\hline Cocaine & & & 31.0 \\
\hline Hallucinogens & & & 4.8 \\
\hline Number of dependencies & 1.52 & 1.56 & \\
\hline
\end{tabular}

Race/ethnicity data were collected for NIH reporting purposes.

scored 0 doesn't apply, 1 applies somewhat, or 2 definitely applies. The possible total scores range from 0 to 40 , with higher scores indicating higher psychopathic traits. In addition to the total score, a two-factor structure was also examined (Hare, 2003; Harpur, Hare, \& Hakstian, 1989). Factor 1 is composed of interpersonal and affective traits (e.g., lack of remorse, grandiosity), whereas Factor 2 is made up of lifestyle and antisocial traits (e.g., poor behavioral controls, impulsivity). Interviews were recorded for reliability assessment and a randomly selected portion of the sample (approximately $10 \%$ ) was double-rated (one-way random-effects model intraclass correlation coefficient $[\mathrm{ICC}]=.91$ for PCL-R total scores, ICC $=.89$ for PCL-R Factor 1 scores, and ICC $=.84$ for PCL-R Factor 2 scores; Shrout \& Fleiss, 1979). Here we used the PCL-R as a continuous measure rather than a dichotomous diagnosis. Using the PCL-R continuously is an acceptable (Hare, 2003) and established (e.g., Decety, Skelly, \& 
Kiehl, 2013; Ermer, Cope, Nyalakanti, Calhoun, \& Kiehl, 2012) use of the instrument. Due to the omission of items due to lack of information (i.e., no history of conditional release, lack of file information, incomplete interview due to release or transfer, etc.), a standard practice in PCL-R scoring, two participants in the sample did not have PCL-R factor scores due to having more than two or three items omitted (for Factors 1 and 2, respectively).

IQ was estimated using the Vocabulary and Matrix Reasoning subtests of the Wechsler Adult Intelligence Scale (WAIS; Ryan, Lopez, \& Werth, 1999; Wechsler, 1997), and reading level was assessed with the Wide Range Achievement Test Word Reading subtest (WRAT-3; Wilkinson, 1993). Two participants in the sample did not complete the WAIS.

Psychiatric and substance use histories were assessed with the Structured Clinical Interview for DSM-IV disorders (SCID; First, Spitzer, Williams, \& Gibbon, 1997) (see Table 2 for correlations among the assessments).

\section{Task}

Participants were shown words and phrases describing moral acts or concepts adapted from Schaich Borg et al. (2011). One hundred stimuli were considered noncontroversial; of these, 50 were classified as negatively valenced (hereafter referred to as wrong: e.g., murder, lying, slavery), and 50 were classified as positively valenced (hereafter referred to as not wrong: e.g., charity, kindness, saving lives). An additional 50 stimuli, classified as morally controversial (e.g., animal testing, prostitution, gun control), were also presented. See Supplemental Material 1 for a list of all stimuli. The stimuli in this task had previously been classified in a publication based on ratings from a healthy undergraduate pilot sample (Schaich Borg et al., 2011). Participants were presented with a stimulus and asked to press one button to indicate that they thought the word or phrase was morally wrong, and another to indicate that they thought the word or phrase was not wrong. Participants were not given any information about what morally wrong meant, although they were told that there were not any right or wrong answers and were given the opportunity to ask questions before and after completing practice stimuli. Immediately after the button was pressed, or after $10 \mathrm{~s}$ if no response was given, a black screen was presented jittered for 1 to $6 \mathrm{~s}$. Participants completed three runs, each consisting of 50 stimuli evenly divided among the stimulus types. The sequence of stimuli within each run was randomized during design; participants completed runs in a counterbalanced order.

To ensure the appropriateness of using this task at $1.5 \mathrm{~T}$ (relative to $3 \mathrm{~T}$ ) MRI and in an incarcerated sample, we replicated the Schaich Borg et al. (2011) sample in a community control sample and the complete forensic sample; consistency between results across our samples and the previous study supported use of the task. See Supplemental Material 2 for details and results from this procedure.

The average word lengths for the included stimuli were 18.3 letters for the wrong condition, 17.4 letters for the not wrong condition, and 16.0 for the controversial condition. A one-way analysis of variance revealed no significant difference in word length between condition $[F(3,193)=0.626, p$ $=.599]$.

\section{Data acquisition}

Participants were scanned using the Mind Research Network's 1.5-T Siemens Avanto mobile MRI scanner stationed at correctional facilities or at the Mind Research Network. The scans were acquired using an echoplanarimaging gradient-echo pulse sequence (parameters: TR 2,000 ms, TE $39 \mathrm{~ms}$, flip angle $75^{\circ}$, FOV $24 \times 24 \mathrm{~cm}, 64 \times$ 64 matrix, 4-mm slice thickness, 27 slices). The task was presented using the E-Prime software (Version 2.0; Psychology Software Tools Inc., Sharpsburg, PA). Behavioral data and eye movements were monitored in realtime to ensure that the participants were performing the task.

\section{Behavioral data analysis}

For noncontroversial items (wrong and not wrong), a correctness value was calculated to determine the proportion of button presses that matched the predetermined classification (i.e., the percentage of not wrong stimuli responded to with a buttonpress indicating not wrong). Correctness and response times for each stimulus type were examined using Pearson's correlations with PCL-R total and factor scores. We then used first-order partial correlations to examine the relationship between each PCL-R Factor, accounting for the shared variance of each factor. Additionally, two-tailed, one-sample $t$ tests were performed to investigate differences in response time between wrong and not wrong, as well as controversial and noncontroversial stimuli. Seven participants were excluded for failing to complete the task. IBM SPSS Statistics 20 was used for all behavioral analyses (IBM, 2011).

A subset of the participants $(n=137)$ were also given a postscan questionnaire asking them to rate each stimulus on moral content, emotional arousal, pleasantness, and how likely people are to agree on whether it is wrong or not wrong. We compared the mean ratings for each stimulus on pleasantness and likeliness to prior categorizations, to make sure these categorizations worked in our sample.

\section{Image preprocessing and analyses}

The imaging data were preprocessed using the SPM software (Wellcome Trust Centre for Neuroimaging, Department of Cognitive Neurology, Cambridge, UK). A multistage 
Table 2 Table of correlations between PCL-R scores and other assessment variables

\begin{tabular}{llllll}
\hline & PCL-R Total & PCL-R Factor 1 & PCL-R Factor 2 & Age & IQ \\
\hline PCL-R Factor 1 & $.82^{* *}$ & & & \\
PCL-R Factor 1 & $.86^{* *}$ & $.50^{* *}$ & & \\
Age & $-.14^{*}$ & .08 & $-.34^{* *}$ & .06 & \\
IQ & -.03 & $.14^{*}$ & $.16^{*}$ & $-.27^{* *}$ & -.06 \\
$\#$ Substance dependencies & $.24^{* *}$ & -.05 & $.33^{* *}$ & & \\
${ }^{*} p<.05,{ }^{* *} p<.01$. & & & & &
\end{tabular}

procedure was used to address the issue of head motion. First, the ArtRepair Toolbox in SPM (Mazaika, Hoeft, Glover, \& Reiss, 2009) was used to identify and remove severe artifacts, defined as time points with greater than $4 \%$ signal change from the global mean signal. Next, head motion was estimated using INRIAlign, an algorithm that is insensitive to eye movements and blood-oxygenation-level-dependent activity (Freire, Roche, \& Mangin, 2002). The ArtRepair Toolbox removes severe motion artifacts but does not account for smaller, more distributed effects of motion. INRIAlign software creates parameters that account for remaining motion and includes it as a variable in the modeling of hemodynamic response. Images were then spatially normalized to the Montreal Neurological Institute template and smoothed with an 8-mm full-width at half-maximum Gaussian smoothing kernel. A high-pass filter removed low-frequency drift at $1 / 128 \mathrm{~Hz}$. This process does not require or include coregistration to a structural scan. Five individuals that were found to have poor image quality after visual inspection of the image masks were removed from the analyses, resulting in a final sample size of $n=237$.

Three conditions of interest (wrong, not wrong, and controversial) were modeled at the first level (single-subject) using a general linear model (GLM). The stimuli were first preclassified as controversial or noncontroversial; within the noncontroversial pool, the stimuli were then classified as not wrong or wrong. Stimuli that were not consistent with the postscan ratings were not included in those conditions. These were determined from the postscan questionnaire results, in which not wrong and wrong items that were not in the top and bottom third of mean pleasantness ratings, respectively, and controversial items not in the bottom third of agreement were found to be inconsistent, resulting in a total of 120 stimuli modeled in the conditions of interest. To model these conditions, vectors of onsets for wrong, not wrong, and controversial stimuli were time-locked to stimulus onset.

Second-level main effect analyses were conducted in which comparisons of the conditions of interest were performed using one-sample, one-tailed $t$ tests. The primary contrasts examined were controversial $>$ noncontroversial, wrong $>$ not wrong, and not wrong $>$ wrong. Noncontroversial was made up of the wrong and not wrong conditions, modeled together. For each contrast, psychopathy- related effects (i.e., total PCL-R scores) were examined in a multiple regression model including covariates of age and IQ $(n=235)$. An additional multiple regression was performed to examine PCL-R Factors 1 and 2, also modeling age and IQ ( $n$ $=233$ ). All regressions were conducted at the voxel level on the basis of the single-subject GLM in SPM.

To test our hypotheses, a priori brain regions of interest (ROIs) were examined. We used anatomical masks generated using the Wake Forest University Pick Atlas in SPM (Maldjian, Laurienti, \& Burdette, 2004; Maldjian, Laurienti, Kraft, \& Burdette, 2003) for the amygdala, ACC, PCC, vAI, TPJ, OFC, and dIPFC (see Supplemental Material 3 for a schematic representation of the masks used). These ROI masks were used to examine each bilateral region. Anatomical ROIs were used, given the large size of clusters in the healthy findings; anatomical ROIs better represent the extents of the clusters activated in the task than do smaller, functionally defined coordinate center spheres. The initial threshold was $p<.05, k=10$. A small-volume correction (SVC) was then applied to determine corrected $p$ values based on the size of each ROI. These results were thresholded at $p<$ .05 with family-wise error rate correction. All imaging analyses were done using SPM.

\section{Results}

\section{Behavioral data (see Table 3 for statistics)}

Item responses Incarcerated participants rated approximately one-half of the stimuli as wrong and one-half as not wrong. However, a significant correlation was apparent between the number of items identified as not wrong and the PCL-R total score for all stimuli. That is, individuals with high psychopathy scores rated more stimuli as being not wrong than did individuals low on psychopathy. This relationship was driven by the PCL-R Factor 2 score (controlling for Factor 1). There was no relationship between item responses and age, IQ, or severity of substance dependence. 
Table 3 Table of behavioral results

\begin{tabular}{|c|c|c|c|c|c|}
\hline & Mean & $\begin{array}{l}\text { Standard } \\
\text { Deviation }\end{array}$ & $\begin{array}{l}\text { Correlation With } \\
\text { PCL-R Total }\end{array}$ & $\begin{array}{l}\text { Partial Correlation } \\
\text { with PCL-R Factor } 1\end{array}$ & $\begin{array}{l}\text { Partial Correlation } \\
\text { With PCL-R Factor } 2\end{array}$ \\
\hline Number of not wrong button presses & 74.70 & 9.11 & $.20^{* * * *}$ & .09 & .11 \\
\hline Number of wrong button presses & 74.28 & 9.44 & $-.19^{* * * *}$ & -.05 & $-.14^{*}$ \\
\hline Correctness, overall & $93 \%$ & 0.11 & $-.15^{*}$ & -.02 & $-.13^{*}$ \\
\hline Correctness, not wrong items & $95 \%$ & 0.12 & -.07 & .02 & -.08 \\
\hline Correctness, wrong items & $91 \%$ & 0.12 & $-.21^{* * * * *}$ & -.06 & $-.16^{*}$ \\
\hline Response time, overall & $2,248 \mathrm{~ms}$ & 472 & .02 & -.02 & .05 \\
\hline Response time, not wrong items & $2,013 \mathrm{~ms}$ & 584 & -.02 & -.01 & -.01 \\
\hline Response time, wrong items & $2,018 \mathrm{~ms}$ & 572 & -.04 & 0 & -.04 \\
\hline Response time, controversial items & $2,507 \mathrm{~ms}$ & 648 & -.06 & -.02 & -.04 \\
\hline Response time, noncontroversial items & $2,016 \mathrm{~ms}$ & 568 & -.03 & -.01 & -.02 \\
\hline
\end{tabular}

The correlations for Factor 1 and Factor 2 scores are first-order partial correlations with variance from the other factor partialed out. ${ }^{*} p<.05,{ }^{* *} p<$ $.01,^{* * *} p<.005,^{* * * * *} p<.001$.

Correctness The PCL-R total score was inversely correlated with overall correctness for wrong but not for not wrong stimuli. In other words, individuals higher in psychopathy were worse at identifying wrong stimuli (moral violations) as wrong. The PCL-R Factor 2 score was correlated with correctness on noncontroversial stimuli and on wrong items after controlling for PCL-R Factor 1; the PCL-R Factor 1 score was not correlated with correctness on any stimulus type.

Response time Response times did not differ between wrong and not wrong stimuli, but they were longer for controversial than for noncontroversial stimuli $(p<$ .001). Response times were not significantly related to PCL-R total or factor scores for any stimulus type. They were also unrelated to age, IQ, or severity of substance dependence.

\section{Imaging}

Comparison of wrong $>$ not wrong stimuli See Table 4 and Fig. 1. In the main ROI analyses, a significant negative relationship between hemodynamic response and PCL-R total score was found in the ACC $(x=0, y=3, z=-9)$. We observed no significant first-order partial correlations between PCL-R factor scores and neural engagement. No significant effects were found in the not wrong $>$ wrong condition.

To better understand the role of psychopathy in wrong and not wrong moral processing, we examined two supplementary contrasts: noncontroversial wrong $>$ baseline and noncontroversial not wrong $>$ baseline. However, no relationship between PCL-R total score and hemodynamic response was found in either contrast (either at the whole-brain level with false discovery rate [FDR] correction, or in the ROIs with SVC).
Comparison of controversial $>$ noncontroversial stimuli See Table 4 and Fig. 2. In the ROI analysis, a significant inverse relationship between hemodynamic response and PCL-R total score was present in both the TPJ ( $x=48, y=-$ $57, z=39)$ and dIPFC $(x=42, y=21, z=36)$. No significant first-order partial correlations emerged between brain activity and PCL-R Factor 1 or 2.

To better understand the contrast of controversial and noncontroversial stimuli, we modeled and examined two supplementary contrasts following the methods already described: controversial $>$ wrong and controversial $>$ not wrong. For Contrast 1, PCL-R was inversely related to hemodynamic engagement in several regions that survived correction for multiple comparisons, using a threshold of $p<.05$ after FDR correction and $k=10$. These regions included the TPJ, dIPFC, and mPFC (see Fig. 3 and Table 5). No activations survived correction for multiple comparisons in Contrast 2. However, following the previous ROI analysis with SVC, both the dIPFC $(t=-3.75)$ and TPJ $(t=-3.88)$ ROIs were significantly inversely related to PCL-R total score.

\section{Discussion}

This study tested the hypotheses that (1) hemodynamic response in the amygdala, ACC, PCC, vAI, and the TPJ would be inversely related to PCL-R score during the processing of wrong versus not wrong moral stimuli; (2) psychopathy would be related to reduced engagement in the OFC, dlPFC, and ACC during not wrong moral processing; and (3) the inverse effects of psychopathy related to controversial moral judgment would be more pronounced in the amygdala, ACC, PCC, vAI, TPJ, OFC, and dIPFC.

We first examined the differences in neural processing of wrong versus not wrong and not wrong versus wrong moral 
Table 4 Region-of-interest (ROI) results for the regression of PCL-R total and factor scores by hemodynamic response

\begin{tabular}{|c|c|c|c|c|c|c|c|c|c|c|c|c|c|}
\hline & \multicolumn{4}{|c|}{ Wrong $>$ Not Wrong } & \multicolumn{4}{|c|}{ Not Wrong > Wrong } & \multicolumn{5}{|c|}{ Controversial > Noncontroversial } \\
\hline & $\begin{array}{ll}x & y\end{array}$ & $z$ & $t$ & $k$ & $x$ & $z$ & $t$ & $k$ & $x$ & $y$ & $z$ & $t$ & $k$ \\
\hline Amygdala & \multicolumn{4}{|l|}{ N.S. } & \multicolumn{4}{|c|}{ Not examined } & \multicolumn{5}{|l|}{ N.S. } \\
\hline Anterior cingulate cortex & 03 & -9 & $-4.05 * *$ & 26 & \multicolumn{4}{|l|}{ N.S. } & \multicolumn{5}{|c|}{ N.S. } \\
\hline Dorsolateral prefrontal cortex & \multicolumn{4}{|c|}{ Not examined } & N.S. & & & & 42 & 21 & 36 & $-4.10 *$ & 137 \\
\hline Orbitofrontal cortex & \multicolumn{4}{|c|}{ Not examined } & \multicolumn{4}{|l|}{ N.S. } & \multicolumn{5}{|l|}{ N.S. } \\
\hline Posterior cingulate cortex & \multicolumn{4}{|l|}{ N.S. } & \multicolumn{4}{|c|}{ Not examined } & \multicolumn{5}{|l|}{ N.S. } \\
\hline Temporoparietal junction & \multicolumn{4}{|l|}{ N.S. } & \multicolumn{4}{|c|}{ Not examined } & 48 & -57 & 39 & $-4.46^{* *}$ & 394 \\
\hline Ventral anterior insula & \multicolumn{4}{|l|}{ N.S. } & \multicolumn{4}{|c|}{ Not examined } & \multicolumn{5}{|l|}{ N.S. } \\
\hline
\end{tabular}

ROIs selected for psychopathy regression based on hypotheses. "N.S." indicates no suprathreshold clusters found during surviving correction for multiple comparisons. "Not examined" indicates that there was no hypothesis regarding that ROI for that contrast. ${ }^{*} p<.05,{ }^{* * *} p<.01$, FWE-corrected.

stimuli by psychopathy. Consistent with our hypothesis, we found a negative relationship between psychopathy score and hemodynamic response in the ACC. Specifically, the inverse engagement peaked in the ventral/rostral ACC, which has a regulatory role involved in the suppression of goal-irrelevant information (Bush et al., 2002; Etkin, Egner, \& Kalisch, 2011). Rostral ACC activity is also related to error detection (Menon, Adleman, White, Glover, \& Reiss, 2001), inappropriate behavioral responses (Kiehl, Liddle, \& Hopfinger, 2000), empathy processes (particularly when thinking about others than oneself; Singer et al., 2004), and reward processes during social cooperation (Rilling et al., 2002). Lower

\section{Beta Values for Anterior Cingulate by PCL- R Total Score}

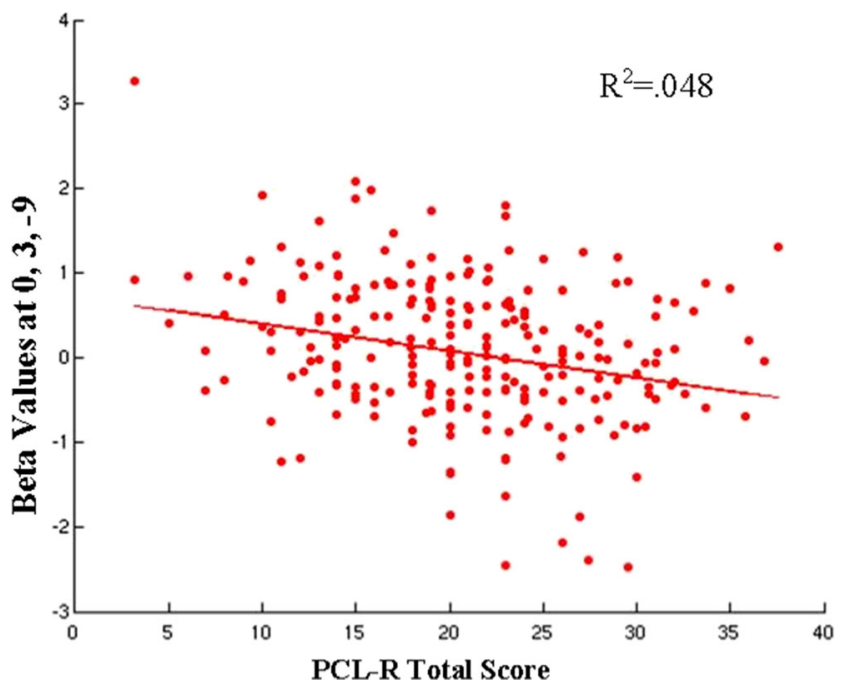

Fig. 1 Plot of the relationships between region-of-interest (ROI) activity and Hare Psychopathy Checklist-Revised (PCL-R) total score for the Wrong $>$ Not Wrong contrast. Lines represent regression functions that control for the variance of age and IQ, rather than best-fit lines for the scatterplots displayed here. Only ROIs with a significant relationship $(p<$ .05 with family-wise error rate correction) are plotted here. Beta values were extracted from the voxel of peak activation found in the smallvolume correction analysis engagement of this region has been found to be related to increased risk for reoffending (Aharoni et al., 2013).

That being said, when examining the underlying conditions, this result was driven by distinct patterns of wrong (negative) and not wrong (positive) moral processing related to psychopathy in this region. Although those low on psychopathy have reduced engagement in the ACC when considering not wrong as compared to wrong moral stimuli (as was also shown by Schaich Borg et al., 2011), those high in psychopathy do not show this same distinction. Therefore, our contrast reflects a reduction in the difference of brain engagement between the two conditions related to psychopathy, suggesting a distinct neural pattern of moral processing.

This is, however, inconsistent with our second hypothesis, in which we expected reduced ACC activity to be associated with psychopathy during not wrong moral processing. In fact, ACC engagement during this condition was positively related to PCL-R total score. Although unexpected, the neural correlates of not wrong moral processing and psychopathy have not previously been examined. This abnormality in not wrong moral processing, although not in the expected direction, may reflect an increased need for the regulatory processes in the ventral/rostral ACC. Further research into this phenomenon is needed.

Next, we examined the association between psychopathy and the brain regions activated during the processing of morally controversial stimuli. As predicted, in response to controversial moral stimuli, an inverse relationship between psychopathy and hemodynamic response was found in the TPJ and dlPFC. The TPJ is recruited during processing of controversial moral stimuli in healthy controls (Schaich Borg et al., 2011) and when attributing intention to others during moral reasoning (Young et al., 2010). Previous work found a negative correlation between TPJ activity and moral severity ratings in psychopaths, but not in nonpsychopaths (Harenski et al., 2010). Furthermore, in females, psychopathic traits were related to reduced TPJ activity overall during the rating of severity of moral violations (Harenski et al., 2014). 
Beta Values for Temporoparietal Junction by PCL-R Total Score

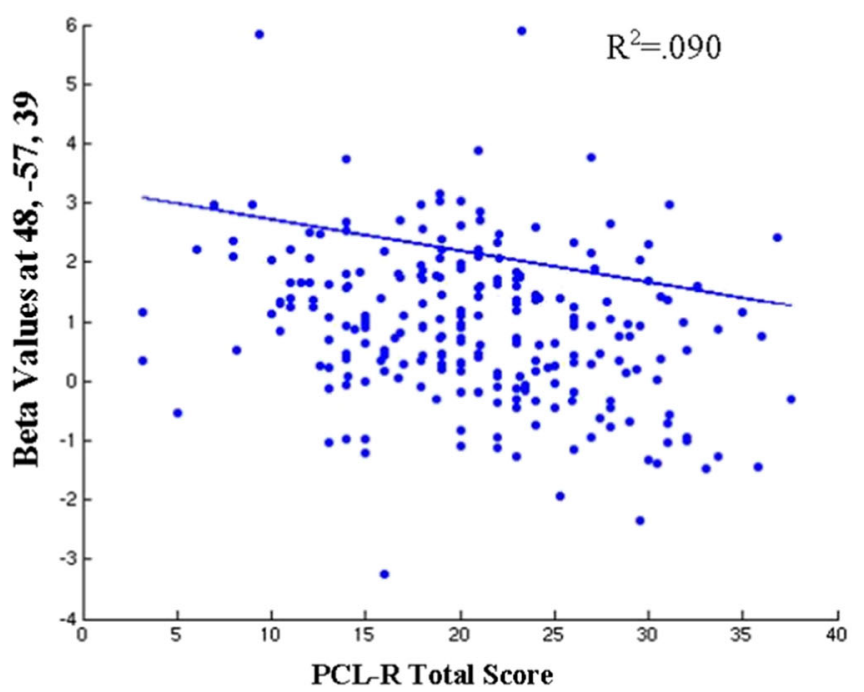

Fig. 2 Plots of the relationships between ROI activity and PCL-R total score for the Controversial > Noncontroversial contrast. Lines represent regression functions that control for the variance of age and IQ, rather than best-fit lines for the scatterplots displayed here. Only ROIs with a

The dIPFC plays an important role in moral judgment as well, being implicated in cognitive control over emotions during dilemmas, abstract reasoning, and generation of aversive emotions (Tassy, Oullier, Cermolacce, \& Wicker, 2009). In previous work, Glenn and colleagues found a positive relationship between dIPFC activity and psychopathy during moral decision making (Glenn, Raine, Schug, Young, \& Hauser, 2009), which the authors have suggested indicates that psychopaths recruit abstract reasoning processes during moral decision making. Our results do not support this conclusion, instead being consistent with other studies that have shown decreased hemodynamic response during moral processing and moral judgment in psychopathy (Glenn, Raine, \& Schug, 2009; Harenski et al., 2010). Together with results from the TPJ, our study indicates that psychopaths do not recruit moral decision making or cognitive neural resources to the same extent that nonpsychopaths and healthy controls do during challenging moral dilemmas. This may or may not indicate a deficit in moral processing; it may simply represent a differential process.

Methodological differences between our study and that of Glenn, Raine, Schug, et al. (2009) should be considered when interpreting the dissimilar results. Here, we used a forensic rather than community sample and our study involved a larger sample ( $n=237$ vs. $n=17)$. Additionally, we investigated morally valenced stimuli composed of words and short phrases as opposed to complex moral personal dilemmas. Finally, the cluster investigated by Glenn, Raine, Schug, et al. was more ventrolateral than the findings observed here, suggesting that we may in fact be investigating areas of the dIPFC with distinct functions.
Beta Values for Dorsolateral Prefrontal Cortex by PCL-R Total Score

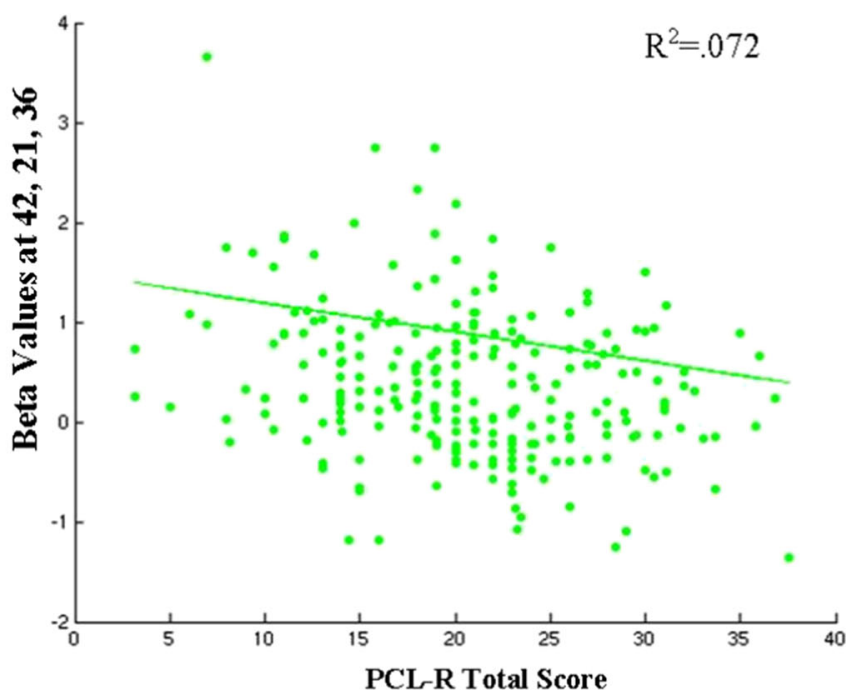

significant relationship ( $p<.05$ with family-wise error rate correction) are plotted here. Beta values were extracted from the voxel of peak activation found in the small-volume correction analysis

Another context in which to interpret these results is that rather than requiring more moral processing, individuals spent more time on controversial stimuli in order to weigh their own immediate reaction with expectations of other people's potentially different opinions. However, participants were instructed to answer whether they thought the stimuli was wrong or not wrong, not what they thought others would think, and that there was no right or wrong answer. Additionally, balancing emotional gut reactions with social influences and rules is an essential part of moral cognition (Moll et al., 2005). Therefore, even if the greater reaction time for controversial than for noncontroversial stimuli reflects this integrative process rather than a unitive moral processing, the findings can still be considered in the context of moral cognition.

A possible concern regarding this comparison is that pooling wrong and not wrong moral stimuli into a noncontroversial comparison results in a non-orthogonal and potentially biased comparison. In order to address that concern, as well as to determine if the difference between controversial and noncontroversial stimuli is driven by moral valence, we also examined the controversial stimuli with wrong and not wrong stimuli separately. In fact, the inverse relationship between PCL-R total score and hemodynamic response was stronger in the comparison of controversial to (noncontroversial) wrong stimuli in both the TPJ and the dIPFC; additionally, engagement of the mPFC, a key moral processing region, was negatively related to PCL-R total score. However, with SVC analysis, engagement of the TPJ and dIPFC negatively related to psychopathy was also 


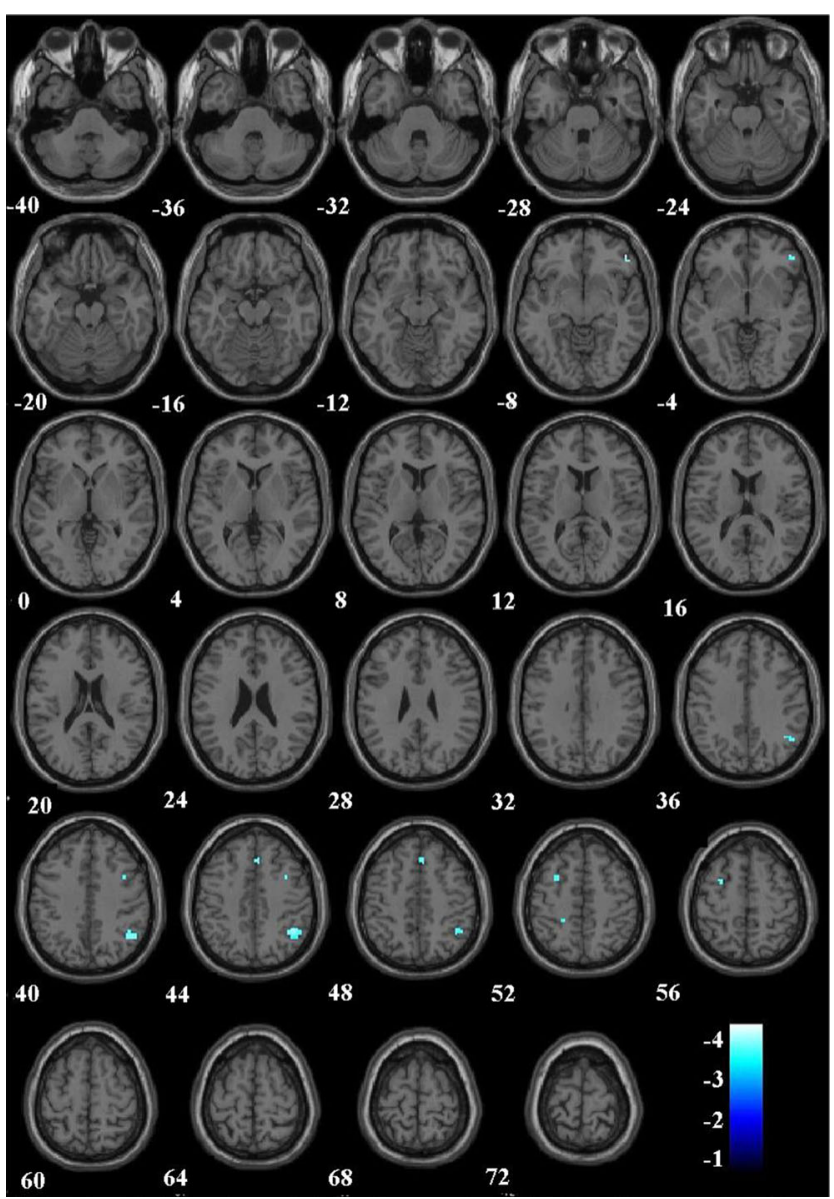

Fig. 3 Results of PCL-R by hemodynamic response regression for the Controversial $>$ Noncontroversial Wrong contrast. The results shown are from whole-brain regression with a false-discovery rate correction at $p<$ .05 and $k=10$. Winter scale colors reflect negative $t$ values

significant in the comparison of controversial to (noncontroversial) not wrong stimuli. This has two-fold importance. First, nonorthogonality does not drive the findings of the TPJ or dIPFC in the controversial > noncontroversial condition. Second, this relationship between psychopathy and neural moral processing is not driven by either the wrong or the not wrong valence of moral stimuli. This may to some extent allay concerns about the subjectivity of the categorization of controversial moral stimuli. Often times, individuals do have a valenced reaction to controversial moral stimuli, particularly when they consider them to be wrong. However, we did not find effects of psychopathy driven by differences in wrong and not wrong valance.

Higher psychopathy scores were also related to poorer performance on the moral processing task. Higher psychopathy total scores were associated with more incorrect responses, specifically by more buttonpresses indicating not wrong in response to wrong stimuli. This effect was driven by antisocial/developmental/lifestyle traits. PCL-R Factor 2 scores were related to fewer correct responses, whereas Factor 1 scores were not significantly related to the behavioral data. This adds to the few studies that have shown effects of psychopathy on moral judgment. The literature is mixed with respect to whether psychopaths perform worse on tasks of moral processing than do nonpsychopaths. Many studies have shown intact moral decision making. However, other work has revealed that psychopaths consider moral personal violations as more permissible than do healthy controls (Cima et al., 2010; Koenigs et al., 2012). The present finding adds to this conclusion by providing evidence that psychopaths may be impaired in distinguishing between right and wrong. Such scientific evidence could have major impacts in legal settings (Aharoni, Funk, Sinnott-Armstrong, \& Gazzaniga, 2008; Morse, 2008).

Given that our behavioral results were driven by PCL-R Factor 2 scores, it may be that antisocial behavior, rather than psychopathy itself, leads to this abnormal pattern of responding. However, there are several reasons to consider these in the context of psychopathy. First, all of the participants had antisocial behavior. In fact, $80 \%-90 \%$ of incarcerated populations meet the criteria for antisocial personality disorder (Hart \& Hare, 1989). PCL-R Factor 2 is also distinct from both antisocial personality disorder and criminality. Its diagnostic criteria include personality rather than behavioral assessments of impulsivity, parasitic orientation, stimulation seeking, and poor behavioral control (Hare, 2003). It also measures life-course-persistent elements of psychopathy, even before criminal behavior begins. PCL-R Factor 2 scores had a large range in our sample (minimum of 2, maximum of 20), despite including criminals with similar levels of offending, indicating that it represents something meaningfully distinct from antisocial behavior and criminality. Finally, the

Table 5 Whole-brain results for controversial > noncontroversial wrong

\begin{tabular}{llllll}
\hline Region & BA & $x$ & $y$ & $z$ & $t$ \\
\hline Dorsolateral prefrontal cortex & 47 & 51 & 39 & -6 & -4.81 \\
Middle frontal cortex & 8 & 6 & 27 & 45 & -4.02 \\
& 6 & -30 & 6 & 54 & -4.37 \\
Supramarginal gyrus/Temporoparietal junction & 40 & 42 & -51 & 45 & -4.42 \\
\hline
\end{tabular}

All regions are significant at $p<.05$ with whole-brain false-discovery rate correction and additionally thresholded at a voxel level of $k=10$. 
neurobiological findings suggest that moral processing is related to both factors of psychopathy and that abnormalities are present in psychopaths. We conducted a supplementary analysis comparing high scorers (i.e., those meeting diagnostic criteria of psychopathy, PCL-R $\geq 30, n=25$ ) and low scorers (PCL-R $<20, n=99$ ), controlling for age and IQ as in the main analysis (Hare, 2003). By examining group differences in ROIs generated from our peak findings using 10-mm-radius spheres and applying SVC, we determined that psychopaths have less engagement of the TPJ, dlPFC, and ACC brain regions during moral processing than nonpsychopaths, consistent with our main findings.

A few limitations need to be considered when interpreting the results described here. We did not include substance dependence as a covariate in our primary analysis. In a supplementary model including substance dependence as a covariate (as quantified by number of SCID substance dependencies), the results were substantively the same as those presented in the main analysis. Thus, we do not believe the present results are impacted by substance use. Another potential concern is that an aspect of linguistic processing, such as word length or frequency, might be driving the results, rather than moral processing. We did not find differences between conditions on word length; however, information on the frequency of these phrases in language is unknown.

Caution should be taken in generalizing the results from this specific task to the moral cognition field as a whole. Different tasks may reflect various aspects of moral judgment that are abnormal to different degrees in psychopathy. For example, many of the negative moral stimuli used here reflect proscriptive morality, or moral guidelines based on rules and negative consequences, whereas those used in "trolley dilemma" tasks are more prescriptive, or abstract and discretionary morality (JanoffBulman, Sheikh, \& Hepp, 2009). This is particularly relevant with psychopathy, considering that psychopaths show a specific lack of sensitivity to punishment (Blair et al., 2004). Contradictions among the results using different moral tasks reflect this concern about differences between the tasks and indicate that additional studies will be needed to accurately generalize the present results to the moral-processing field as a whole.

Prior studies have reported psychopathy-related abnormalities in the amygdala when processing negative moral stimuli (Blair, 2007; Glenn, Raine, \& Schug, 2009). We did not find that activity in the amygdala was related to psychopathy during the processing of wrong linguistic moral stimuli. In our task, participants were explicitly directed to consider the morality of the linguistic stimuli. Studies have shown that psychopathy-associated deficits in amygdala function are reduced when attention is directed at the salient stimulus of interest (Larson et al., 2013; Newman, 1998). Also, it may be that psychopathy-related amygdala effects are most prominent during implicit tasks or tasks that automatically engage the amygdala (i.e., startle stimuli, salient environmental stimuli, etc.). In another study of explicit visual moral judgment, the investigators failed to find any relationship between psychopathy and moral judgment in the amygdala (Harenski et al., 2010).

In summary, here we have replicated existing results of a moral decision-making task and extended that work using a forensic sample. Psychopathic traits were related to brain abnormalities in moral-processing regions during the processing of controversial moral stimuli. We also found differential patterns of wrong and not wrong moral processing that were associated with psychopathy. This work helps to elucidate the neurobiological basis of impairments in moral processing in psychopathy.

Author note This work was supported by the National Institute of Mental Health (Grant No. R01MH070539 to K.A.K.) and the National Institute on Drug Abuse (Grant Nos. R01DA026964 and R01DA026505 to K.A.K.). The content is solely the responsibility of the authors and does not necessarily represent the official views of the National Institutes of Health. The authors are not aware of any conflicts of interest. Portions of these data were presented at the annual meeting of the Society for the Scientific Study of Psychopathy (June 2013) and submitted as a thesis for partial fulfillment of the Master of Science degree, by S.J.F. at the University of New Mexico (November 2014).

\section{References}

Aharoni, E., Funk, C., Sinnott-Armstrong, W., \& Gazzaniga, M. (2008). Can neurological evidence help courts assess criminal responsibility? Lessons from law and neuroscience. Annals of the New York Academy of Sciences, 1124, 145-160. doi:10.1196/annals.1440.007

Aharoni, E., Sinnott-Armstrong, W., \& Kiehl, K. A. (2012). Can psychopathic offenders discern moral wrongs? A new look at the moral/ conventional distinction. Journal of Abnormal Psychology, 121, 484-497. doi:10.1037/a0024796

Aharoni, E., Vincent, G. M., Harenski, C. L., Calhoun, V. D., SinnottArmstrong, W., Gazzaniga, M. S., \& Kiehl, K. A. (2013). Neuroprediction of future rearrest. Proceedings of the National Academy of Sciences, 110, 6223-6228.

Alterman, A. A., Cacciola, J. S., \& Rutherford, M. J. (1993). Reliability of the revised psychopathy checklist in substance abuse patients. Psychological Assessment, 5, 442-448.

Anderson, D. A. (2012). The cost of crime. Foundations and Trends in Microeconomics, 7, 209-265.

Blair, R. J. R. (1995). A cognitive developmental approach to mortality: Investigating the psychopath. Cognition, 57, 1-29. doi:10.1016 /0010-0277(95)00676-P

Blair, R. J. R. (2007). The amygdala and ventromedial prefrontal cortex in morality and psychopathy. Trends in Cognitive Sciences, 11, 387392. doi:10.1016/j.tics.2007.07.003

Blair, R. J. R., Mitchell, D. G. V., Leonard, A., Budhani, S., Peschardt, K. S., \& Newman, C. (2004). Passive avoidance learning in individuals with psychopathy: Modulation by reward but not by punishment. Personality and Individual Differences, 37, 1179-1192. doi:10.1016 /j.paid.2003.12.001 
Boccardi, M., Frisoni, G. B., Hare, R. D., Cavedo, E., Najt, P., Pievani, M.,...Repo-Tiihonen, E. (2011). Cortex and amygdala morphology in psychopathy. Psychiatry Research: Neuroimaging, 193, 85-92.

Bush, G., Vogt, B. A., Holmes, J., Dale, A. M., Greve, D., Jenike, M. A., \& Rosen, B. R. (2002). Dorsal anterior cingulate cortex: A role in reward-based decision making. Proceedings of the National Academy of Sciences, 99, 523-528. doi:10.1073/pnas.012470999

Caldwell, M. F., McCormick, D. J., Umstead, D., \& Van Rybroek, G. J. (2007). Evidence of treatment progress and therapeutic outcomes among adolescents with psychopathic features. Criminal Justice and Behavior, 34, 573-587. doi:10.1177/0093854806297511

Chang, L. J., Yarkoni, T., Khaw, M. W., \& Sanfey, A. G. (2013). Decoding the role of the insula in human cognition: Functional parcellation and large-scale reverse inference. Cerebral Cortex, 23, 739-749. doi:10.1093/cercor/bhs065

Cima, M., Tonnaer, F., \& Hauser, M. D. (2010). Psychopaths know right from wrong but don't care. Social Cognitive and Affective Neuroscience, 5, 59-67. doi:10.1093/scan/nsp051

Cleckley, H. (1976). The mask of sanity: An attempt to clarify some issues about the so-called psychopathic personality. St. Louis, MO: Mosby.

de Oliveira-Souza, R., Hare, R. D., Bramati, I. E., Garrido, G. J., Ignacio, F. A., Tovar-Moll, F., \& Moll, J. (2008). Psychopathy as a disorder of the moral brain: Fronto-temporo-limbic grey matter reductions demonstrated by voxel-based morphometry. NeuroImage, 40, 1202-1213. doi:10.1016/j.neuroimage.2007.12.054

Decety, J., Skelly, L. R., \& Kiehl, K. A. (2013). Brain response to empathy-eliciting scenarios involving pain in incarcerated individuals with psychopathy. JAMA Psychiatry, 70, 638-645.

Ermer, E., Cope, L. M., Nyalakanti, P. K., Calhoun, V. D., \& Kiehl, K. A. (2012). Aberrant paralimbic gray matter in criminal psychopathy. Journal of Abnormal Psychology, 121, 649-658. doi:10.1037 /a0026371

Ermer, E., Guerin, S. A., Cosmides, L., Tooby, J., \& Miller, M. B. (2006). Theory of mind broad and narrow: Reasoning about social exchange engages ToM areas, precautionary reasoning does not. Social Neuroscience, 1, 196-219. doi:10.1080/17470910600989771

Ermer, E., \& Kiehl, K. A. (2010). Psychopaths are impaired in social exchange and precautionary reasoning. Psychological Science, 21, 1399-1405. doi:10.1177/0956797610384148

Etkin, A., Egner, T., \& Kalisch, R. (2011). Emotional processing in anterior cingulate and medial prefrontal cortex. Trends in Cognitive Sciences, 15, 85-93. doi:10.1016/j.tics.2010.11.004

First, M. B., Spitzer, R. L., Williams, J. B. W., \& Gibbon, M. (1997). Structured clinical interview for DSM-IV (SCID). Washington, DC: American Psychiatric Association.

Fletcher, P. C., Happe, F., Frith, U., Baker, S. C., Dolan, R. J., Frackowiak, R. S. J., \& Frith, C. D. (1995). Other minds in the brain: A functional imaging study of theory of mind in story comprehension. Cognition, 57, 109-128. doi:10.1016/0010-0277(95)00692-R

Freire, L., Roche, A., \& Mangin, J.-F. (2002). What is the best similarity measure for motion correction in fMRI time series? IEEE Transactions on Medical Imaging, 21, 470-484.

Glenn, A. L., Raine, A., \& Schug, R. A. (2009). The neural correlates of moral decision-making in psychopathy. Molecular Psychiatry, 14, 5-6. doi:10.1038/mp.2008.104

Glenn, A. L., Raine, A., Schug, R. A., Young, L., \& Hauser, M. (2009). Increased DLPFC activity during moral decision-making in psychopathy. Molecular Psychiatry, 14, 909-911. doi:10.1038 /mp.2009.76

Greene, J. D., Sommerville, R. B., Nystrom, L. E., Darley, J. M., \& Cohen, J. D. (2001). An fMRI investigation of emotional engagement in moral judgment. Science, 293, 2105-2108. doi:10.1126 /science.1062872

Hare, R. D. (2003). Manual for the Hare Psychopathy Checklist-Revised (2nd ed.). Toronto, Canada: Multi-Health Systems.
Harenski, C. L., Edwards, B. G., Harenski, K. A., \& Kiehl, K. A. (2014). Neural correlates of moral and non-moral emotion in female psychopathy. Frontiers in Human Neuroscience, 8, 741. doi:10.3389 /fnhum.2014.00741

Harenski, C. L., Harenski, K. A., Shane, M. S., \& Kiehl, K. A. (2010). Aberrant neural processing of moral violations in criminal psychopaths. Journal of Abnormal Psychology, 119, 863-874. doi:10.1037 /a0020979

Harpur, T. J., Hare, R. D., \& Hakstian, A. R. (1989). Two-factor conceptualization of psychopathy: Construct validity and assessment implications. Psychological Assessment, 1, 6-17. doi:10.1037/10403590.1.1.6

Hart, S. D., \& Hare, R. D. (1989). Discriminant validity of the Psychopathy Checklist in a forensic psychiatric population. Psychological Assessment, 1, 211-218. doi:10.1037/10403590.1.3.211

IBM. (2011). IBM SPSS Statistics for Windows (Version 20.0). Armonk, NY: IBM Corp.

Janoff-Bulman, R., Sheikh, S., \& Hepp, S. (2009). Proscriptive versus prescriptive morality: Two faces of moral regulation. Journal of Personality and Social Psychology, 96, 521-537. doi:10.1037 /a0013779

Johnson, M. K., Raye, C. L., Mitchell, K. J., Touryan, S. R., Greene, E. J., \& Nolen-Hoeksema, S. (2006). Dissociating medial frontal and posterior cingulate activity during self-reflection. Social Cognitive and Affective Neuroscience, 1, 56-64. doi:10.1093/scan/ns1004

Juárez, M., Kiehl, K. A., \& Calhoun, V. D. (2013). Intrinsic limbic and paralimbic networks are associated with criminal psychopathy. Human Brain Mapping, 34, 1921-1930.

Kiehl, K. A. (2006). A cognitive neuroscience perspective on psychopathy: Evidence for paralimbic system dysfunction. Psychiatry Research, 142, 107-128. doi:10.1016/j.psychres.2005.09.013

Kiehl, K. A. (2014). The psychopath whisperer: The science of those without conscience. New York, NY: Crown.

Kiehl, K. A., \& Hoffman, M. B. (2011). The criminal psychopath: History, neuroscience, treatment, and economics. Jurimetrics, 51, 355-397.

Kiehl, K. A., Liddle, P. F., \& Hopfinger, J. B. (2000). Error processing and the rostral anterior cingulate: An event-related fMRI study. Psychophysiology, 37, 216-223. doi:10.1017/S0048577200990231

Kiehl, K. A., Smith, A. M., Hare, R. D., Mendrek, A., Forster, B. B., Brink, J., \& Liddle, P. F. (2001). Limbic abnormalities in affective processing by criminal psychopaths as revealed by functional magnetic resonance imaging. Biological Psychiatry, 50, 677-684.

Koenigs, M., Kruepke, M., Zeier, J., \& Newman, J. P. (2012). Utilitarian moral judgment in psychopathy. Social Cognitive and Affective Neuroscience, 7, 708-714. doi:10.1093/scan/nsr048

Larson, C. L., Baskin-Sommers, A. R., Stout, D. M., Balderston, N. L., Curtin, J. J., Schultz, D. H.,... Newman, J. P. (2013). The interplay of attention and emotion: top-down attention modulates amygdala activation in psychopathy. Cognitive, Affective, \& Behavioral Neuroscience, 13, 757-770. doi:10.3758/s13415-013-0172-8

Ly, M., Motzkin, J. C., Philippi, C. L., Kirk, G. R., Newman, J. P., Kiehl, K. A., \& Koenigs, M. (2012). Cortical thinning in psychopathy. American Journal of Psychiatry, 169, 743-749. doi:10.1176/appi. ajp.2012.11111627

Ma, Y. N., Wang, C. B., \& Han, S. H. (2011). Neural responses to perceived pain in others predict real-life monetary donations in different socioeconomic contexts. NeuroImage, 57, 1273-1280. doi:10.1016 /j.neuroimage.2011.05.003

Maldjian, J. A., Laurienti, P. J., \& Burdette, J. H. (2004). Precentral gyrus discrepancy in electronic versions of the Talairach atlas. NeuroImage, 21, 450-455.

Maldjian, J. A., Laurienti, P. J., Kraft, R. A., \& Burdette, J. H. (2003). An automated method for neuroanatomic and cytoarchitectonic atlas- 
based interrogation of fMRI data sets. NeuroImage, 19, 1233-1239. doi:10.1016/S1053-8119(03)00169-1

Mazaika, P. K., Hoeft, F., Glover, G. H., \& Reiss, A. L. (2009). Methods and software for fMRI analysis of clinical subjects. NeuroImage, 47, S58.

Menon, V., Adleman, N. E., White, C. D., Glover, G. H., \& Reiss, A. L. (2001). Error-related brain activation during a Go/NoGo response inhibition task. Human Brain Mapping, 12, 131-143. doi:10.1002 /1097-0193(200103)12:3<131::aid-hbm1010>3.0.co;2-c

Moll, J., de Oliveira-Souza, R., Moll, F. T., Ignácio, F. A., Bramati, I. E., Caparelli-Dáquer, E. M., \& Eslinger, P. J. (2005). The moral affiliations of disgust: A functional MRI study. Cognitive and Behavioral Neurology, 18, 68-78.

Morse, S. J. (2008). Psychopathy and criminal responsibility. Neuroethics, 1, 205-212.

Newman, J. P. (1998). Psychopathic behavior: An information processing perspective. In D. J. Cooke, A. E. Forth, \& R. D. Hare (Eds.), Psychopathy: Theory, research and implications for society (pp. 81-104). Dordrecht, The Netherlands: Kluwer.

Ochsner, K. N., \& Gross, J. J. (2005). The cognitive control of emotion. Trends in Cognitive Sciences, 9, 242-249. doi:10.1016/j. tics.2005.03.010

O'Kane, A., Fawcett, D., \& Blackburn, R. (1996). Psychopathy and moral reasoning: Comparison of two classifications. Personality and Individual Differences, 20, 505-514.

Price, J. L. (2003). Comparative aspects of amygdala connectivity. Annals of the New York Academy of Sciences, 985, 50-58.

Rilling, J. K., Glenn, A. L., Jairam, M. R., Pagnoni, G., Goldsmith, D. R., Elfenbein, H. A., \& Lilienfeld, S. O. (2007). Neural correlates of social cooperation and non-cooperation as a function of psychopathy. Biological Psychiatry, 61, 1260-1271. doi:10.1016/j. biopsych.2006.07.021

Rilling, J. K., Goldsmith, D. R., Glenn, A. L., Jairam, M. R., Elfenbein, H. A., Dagenais, J. E.,...Pagnoni, G. (2008). The neural correlates of the affective response to unreciprocated cooperation. Neuropsychologia , 46, 1256-1266. doi:10.1016/j. neuropsychologia.2007.11.033

Rilling, J. K., Gutman, D. A., Zeh, T. R., Pagnoni, G., Berns, G. S., \& Kilts, C. D. (2002). A neural basis for social cooperation. Neuron, 35, 395-405. doi:10.1016/s0896-6273(02)00755-9
Ryan, J. J., Lopez, S. J., \& Werth, T. R. (1999). Development and preliminary validation of a Satz-Mogel short form of the WAIS-III in a sample of persons with substance abuse disorders. International Journal of Neuroscience, 98, 131-140. doi:10.3109 /00207459908994796

Schaich Borg, J., Sinnott-Armstrong, W., Calhoun, V. D., \& Kiehl, K. A. (2011). Neural basis of moral verdict and moral deliberation. Social Neuroscience, 6, 398-413. doi:10.1080/17470919.2011.559363

Shrout, P. E., \& Fleiss, J. L. (1979). Intraclass correlations: Uses in assessing rater reliability. Psychological Bulletin, 86, 420-428. doi:10.1037/0033-2909.86.2.420

Simon, B., Holzberg, J. D., \& Unger, J. F. (1951). A study of judgment in the psychopathic personality. Psychiatric Quarterly, 25, 132-150.

Singer, T., Seymour, B., O’Doherty, J., Kaube, H., Dolan, R. J., \& Frith, C. D. (2004). Empathy for pain involves the affective but not sensory components of pain. Science, 303, 1157-1162.

Tassy, S., Oullier, O., Cermolacce, M., \& Wicker, B. (2009). Do psychopathic patients use their DLPFC when making decisions in moral dilemmas and quest. Molecular Psychiatry, 14, 908-909.

Tiihonen, J., Rossi, R., Laakso, M. P., Hodgins, S., Testa, C., Perez, J.,... Frisoni, G. B. (2008). Brain anatomy of persistent violent offenders: More rather than less. Psychiatry Research, 163, 201-212. doi:10.1016/j.pscychresns.2007.08.012

Wechsler, D. (1997). WAIS-III: Administration and scoring manual: Wechsler Adult Intelligence Scale. New York, NY: Psychological Corp.

Wilkinson, G. (1993). WRAT-3 Wide Range Achievement Test. Wilmington, DE: Wide Range, Inc.

Yang, Y., Raine, A., Narr, K. L., Colletti, P., \& Toga, A. W. (2009). Localization of deformations within the amygdala in individuals with psychopathy. Archives of General Psychiatry, 66, 986-994. doi:10.1001/archgenpsychiatry.2009.110

Young, L., Camprodon, J. A., Hauser, M., Pascual-Leone, A., \& Saxe, R. (2010). Disruption of the right temporoparietal junction with transcranial magnetic stimulation reduces the role of beliefs in moral judgments. Proceedings of the National Academy of Sciences, 107, 6753-6758. doi:10.1073/pnas.0914826107

Young, L., Koenigs, M., Kruepke, M., \& Newman, J. P. (2012). Psychopathy increases perceived moral permissibility of accidents. Journal of Abnormal Psychology, 121, 659-667. doi:10.1037 /a0027489 